\title{
A CASE OF SUB-PHRENIC ABSCESS AND EMPYEMA.
}

\author{
By H. V. MORLOCK, M.C., M.D., M.R.C.P.
}

(Physician, Miller General Hospital, City of London Hospital for Diseases of the Heart and Lungs, and O.P. Dept., Hampstead General Hospital.) \section{history:-}

A male, aged 53 years, was seen in consultation and gave the following

Seven days previously he had noticed "some rheumatism" in the right shoulder and neck. He had, however, continued at work and after four days the pain had ceased, but on the following day he noticed that he was hot, feverish and languid and that he had developed pain in the right chest. He then went to bed, where he had remained, and his symptoms had persisted; but no other symptoms had developed.

At the time of my examination I noted that he was a well-covered, slightlyflushed man showing no apparent distress. The cranial nerves, tongue, teeth, throat and neck presented nothing abnormal. The heart, as regards size and position and sounds, was normal. Chest: right side moved less than left; vocal fremitus diminished below the nipple in front and below the eighth rib behind on the right side; over this same area the percussion note was dull, the breath sounds were absent as also vocal resonance; on the left side, nothing abnormal discovered. Abdomen: right upper quadrant held rather rigid, with some tenderness, otherwise normal.

Central nervous system and urine normal; rectal examination negative.

Temp. I02 ${ }^{\circ} \mathrm{F} . ;$ pulse 100 and resp. 30 per minute.

\section{Commentary.}

The above history and physical signs caused the following various diagnoses to be considered :-

(a) Pneumonia ;

(b) Abscess;

(c) Pulmonary collapse due to a new growth, and

(d) Fluid effusion (serous or purulent).

As regards pneumonia, the patient was not distressed; he had no cough, and the percussion note was so dull as to suggest fluid rather than consolidation.

Against the diagnosis of abscess was the absence of cough and the absence of sputum.

Against the diagnosis of pulmonary collapse was the fact that there was no mediastinal displacement towards the lesion. 
Empyema was the diagnosis that had been previously made, but there was an unusual symptom and an unusual sign for this diagnosis, namely, the onset with "rheumatic pain" in the right shoulder and the definite tenderness and rigidity of the right upper quadrant of the abdomen.

It is well known that irritation of the peritoneal surface of the diaphragm will give rise to pain in the shoulder and neck. One encounters this symptom in perforated peptic ulcer and in a ruptured ectopic gestation. Hence in this case the symptom raised the suspicion of a sub-diaphragmatic lesion, a suspicion which gained support from the tenderness in the right upper abdominal quadrant, for although an empyema often causes referred abdominal pain it is unusual for there to be definite abdominal tenderness and rigidity.

The possibility of a sub-phrenic abscess having been raised, the patient was re-questioned regarding the onset of the illness and the following additional facts were elicited :-

Twelve days before the onset of the "rheumatic pain" in the right shoulder, he had had a sudden attack of abdominal pain, which had not been accompanied by any serious shock, but which was sufficiently severe to send him to bed and keep him there for four days. During this time there had been some flatulence but no vomiting, and the bowels had acted normally. At the end of the four days he had returned to business although not feeling very well, but he was able to carry on for eight days before he had noticed the "rheumatic pain" in the right shoulder and neck.

This additional history made a sub-phrenic abscess the most probable diagnosis.

Was it possible to obtain further clinical evidence in support of this diagnosis? Usually in sub-phrenic abscesses there is a gas bubble under the dome of the diaphragm and lying above the fluid in the abscess. This gas bubble will change its position and remain uppermost, varying with the position which the patient assumes, hence the physical signs in the chest vary with change of position.

The physical signs reported above had been elicited with the patient sitting upright, that is, with the gas bubble under the highest point of the dome of the diaphragm; the patient was therefore laid on his left side when the percussion note over the lower part of the right axilla became more resonant. Thereafter he was made to lie on his face and the percussion note at the right base, which had previously been dull, now became resonant.

These findings made the diagnosis of a sub-phrenic abscess almost certain. The only other condition which might give rise to similar physical signs which vary with change in position is a hydro-pneumothorax; but this would be accompanied by a succussion splash which was absent in this case.

To confirm beyond doubt the diagnosis of sub-phrenic abscess, and to ascertain the most suitable position for the incision at the operation for drainage, it was necessary to take a skiagram. It had to be remembered, however, that if the skiagram, as is customary, is taken with the patient lying down the gas bubble 
below the diaphragm would not be apparent because, the gas bubble having floated upwards to the posterior surface of the diaphragm and the fluid having sunk below, the rays passing downwards through the patient would pass through a layer of fluid and gas and thus fail to show a gas bubble. If, however, the patient is $\mathrm{X}$-rayed in the upright position, the rays pass through the gas bubble with the layer of fluid below it and thus the gas bubble is seen clearly on the skiagram. Therefore, in this case the patient was ordered to be X-rayed in the erect position, and in addition a lateral skiagram was requested in order to ascertain the anteroposterior position of the abscess.

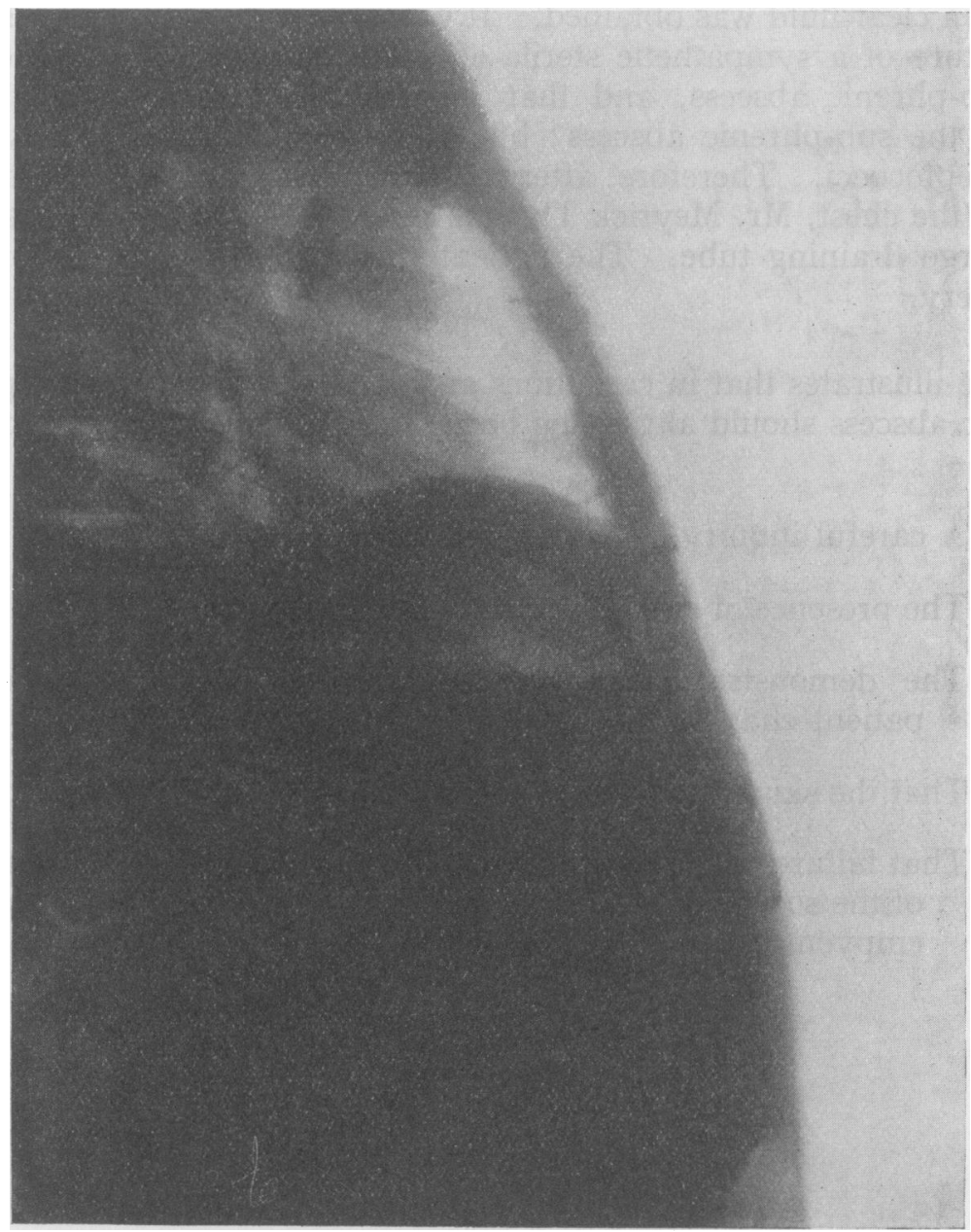

FIG. 1.-Skiagram taken with patient in lateral position showing gas bubble and fluid level below diaphragm.

Fig. I demonstrates the gas bubble in the skiagram taken in lateral position. A little fluid in the pleural cavity was observed in anteroposterior view (not reproduced). 
The diagnosis was thus confirmed and an operation was decided upon. As the abscess extended the whole anteroposterior length of the diaphragm, Mr. Meyrick Thomas decided to drain the abscess posteriorly, because this site would afford the best drainage when the patient assumed the usual position in bed, namely, lying on the back; he therefore removed the tenth rib posteriorly and opened the abscess extrapleurally. Twenty ounces of pus were evacuated and a large drainage tube inserted.

In spite of free drainage during the next ten days, the patient's temperature failed to fall and the signs of fluid in the right pleura increased. The chest was explored and a clear fluid was obtained. It was at first thought that this fluid might be of the nature of a sympathetic sterile effusion, which not uncommonly accompanies a sub-phrenic abscess, and that the continued temperature was due to pocketing of the sub-phrenic abscess; but on culture the effusion gave a profuse growth of streptococci. Therefore, after a preliminary aspiration of fifty-six ounces of fluid from the chest, Mr. Meyrick Thomas resected the eighth rib posteriorly and inserted a large draining tube. The patient then proceeded to make an uninterrupted recovery.

This case illustrates that in conditions at the base of the lung, the possibility of a sub-phrenic abscess should always be borne in mind and that attention should be directed to:-

I. A careful inquiry as to previous abdominal pain.

2. The presence of pain in the shoulder and neck.

3. The demonstration of the alteration of physical signs when the patient changes his position.

4. That the skiagram or the screening must be done with the patient erect.

5. That failure of the patient's condition to improve on adequate drainage of the sub-phrenic abscess arouses the suspicion of an accompanying empyema. 